\title{
Neuropsychological Commentary on Effective Speaking Ability of Doctors
}

\section{Madhav Gajananrao Raje*}

Consultant Psychiatrist and Psychotherapist, Mahakali Clinics, Ayodhya Nagar, Nagpur, India

*Corresponding Author: Madhav Gajananrao Raje, Consultant Psychiatrist and Psychotherapist, Mahakali Clinics, Ayodhya Nagar, Nagpur, India.
Received: September 16, 2021

Published: October 20, 2021

(C) All rights are reserved by Madhav

Gajananrao Raje.

\begin{abstract}
Doctors across the globe undermine the need to develop speaking ability despite awareness. This behavior can be explained on the basis of theory of lateralization; whereby they focus unreasonably on intellectual reasoning rather than emotional accommodativeness. Focus on intellectual reasoning has over the years become an ingrained habit of medical fraternity; creating an invisible wall in between them and patients.

Many authors have explored this area of ineffective communication in between doctor and patient. But medical fraternity's habit could not be reversed efficaciously; may be because of lack of neuro-bio-psychological evidence base explanation.

Neuroscience and psychological perspective underlying ineffective speaking ability of doctors is addressed in this article. This article discusses methods to help doctors change their psychological perspective with due consideration to neuroscience and facilitate to adapt to skills of speaking ability. It explains how to change older thinking pattern to newer thinking pattern and evolve from traditional approach to holistic approach of treatment i.e. 'patient-centered- treatment', a reformed modality of treatment leading to success in career.
\end{abstract}

Keywords: Theory of Lateralization; Patient Centered Communication; Holistic Treatment Approach; Breaking Bad News (BBN); Speaking Ability of Doctor; Evolution of Doctors; Biological Faith; Neuropsychological Commentary; Holistic Doctor; Neuroscience of Unlearning

\section{Introduction}

Patients are emotionally vulnerable when approach medical doctor. Their up regulated emotional brain down regulate their pre-frontal lobe (PFC) and left hemisphere (Theory of Lateralization). This neurobiology gets reflected through their behavior. Example of such behavior could be seen in their urgency of meeting doctor, impulsivity, restlessness, confusion, helplessness or frustration, offensive stand or stand to surrender, etc. Let's take freedom to name this scenario intellectually laded-emotional behav- ior' of patient/relatives. Meaning a person behaves emotionally but his behavior is based on one's own intellectualization/rationalization. This description is largely an identical picture of any patient or care-taker or relative of patient.

Medical fraternity while responding to such situation experiences tangible/intangible stress and thereby unknowingly or knowingly adapts opposite stand which is reflected through their psycho-biological behavioral reactions [1]. Involuntarily there 
by, doctors set aside emotional overtone and relies largely on left hemisphere that encompasses the function of thinking, reasoning, logical deduction, problem solving, anticipation, making decisions, planning and execution. Such overtone of left hemisphere sublimes the ability to visualize, reciprocate, empathize or sympathize, attitude to build faith, out of box thinking which is recognized as gifts of right hemisphere to humanity [2].

Thus, one understands that doctor and patient have contrasting psycho-biological perspectives when meet each other. Patient's perspective about doctor's job is built by their right hemisphere that is based on emotionally laded expectations [2,3]. Patients want doctors to understand their illness and pass on this understanding to them while undergoing treatment. They want to be a partner in defeating their illness though a passive partner [4]. Alignment of patient's expectations and that of treating physician's behavior is a need of hour [4]; which is possible through "patient-centered communication", a term coined long ago by C.L. Bylund., et al [5].

Lack of awareness of doctors about such expectations of patients could build invisible wall in between them. Doctors remain un-aware of such demands because of their less developed social intelligence as against their semantic intelligence. However, only doctor/s can break open this wall because of their relatively better position to adapt to stressful situations as a problem solver. How to deal with such "intellectually laded-emotionally behavior"? 'Speaking ability' is the reply to such behavior. According to theorization of Austin and Searle, communication or speech is a "social activity". To execute it effectively it is imperative for speaker to become social. That means speaker needs some degree of command over language which is an instrument to be social [6].

Speaking ability includes non-verbal as well as verbal components. Verbal component is largely handled by left hemisphere and non-verbal component is largely handled by right hemisphere. Both hemispheres of doctor need to work together, complementing each other with similar proficiency to harness speaking ability. For the sake of ease of expression and comprehension let's call henceforth the capacity of left hemisphere 'ability' and ability of right hemisphere as 'skill'. So, to summarize, let's say all doctors essentially need 'ability' to evaluate patient as well as 'skill' to communicate findings of evaluation to patient. Together let's call it "skilled ability". In alignment with this thought Jennifer Fong Ha (2010) men- tioned conversation in between patient and doctor plays essential role to make treatment wholesome and effective [7].

\section{Need of speaking ability}

25 year old son of my patient once asked me if he should continue or change dermatologist for want of relief even after 3 month's treatment. While showing me pathologist's report he further said that pathologist after listening the same question told him that he is suffering from an unusual skin disease that requires longer time for relief. So, he must not get disheartened and should not change his dermatologist. I spoke to help him to reduce his worries. This scenario illustrates that non-clinical doctor; clinical doctor and a doctor with 'third person's view' too need speaking ability.

My son once phoned me when I was counseling a patient. I picked up the phone and talked with him requesting the patient to wait for a while. On completion of telephonic talk before resuming to counsel I explained the patient that law of priority guides me to prioritize my family members. Further I asked for forgiveness and apology for interruption and assured him about my dedication to serve him. This dialogue was executed in anticipation to deal with patient's silent disapproval.

These two paragraphs adequately highlight the need of speaking ability for every doctor or medical professional.

These examples also indicate that speaking ability is a composite entity which essentially comprises of psycho-social-biological components [6].

Following commentary aims to include psycho-bio-social-philosophical aspects of communication.

Objectives of development of speaking ability or verbal communication

\section{A. Self centered objectives}

1. Re-create self identity: Self identity means perception of one' own self. 'Self' includes perception of one's own characteristics which help one to describe oneself.

Admission to medical school itself recreates identity of any high-school passed adolescent. Before admission to medical school s/he identifies with common masses/all 
adolescents but after becoming a medical student her/his identity changes. Professional activity of medical graduate reinforces this identity. But usefulness of this identity of being doctor can be perceived only when "spoken words" of patients spread around. That means patient's word of mouth re-creates doctor's identity in real world.

Doctors usually expect themselves to speak knowledge based medical information. Hence doctors may assume need of harnessing speaking ability a little less important against their ability to pass on sematic knowledge. But if doctor realizes that speaking doesn't mean conveying of medical information only [6] but it comprises of all four aspects mentioned earlier; then only doctor would brace up to enhance his or her speaking ability. When this four pronged ability (Psycho-bio-social and philosophical) is adapted then identity of doctor gets existentially perceive by all concerned.

\section{Evolution of doctors}

a) Evolution from "doctor" to "holistic doctor": [7-9] Holistic modern medicine doctor (One with holistic approach to treat) sees the patient as a complete individual not just a victim of illness. S/He tends to treat not only the disease but the patient as a whole. He thinks, comprehends and acts there upon without subjective point of view and differently than that of any other doctor with professional-cum-commercial point of view.

Holistic doctor considers patient's psychological makeup, financial limitations, family/social support, and life as a whole. He influences patient's life beyond his areas of medical expertise through his verbal/nonverbal communication based on his ideology. His ideology helps him consider a human being as whole; a bundle of feelings, thoughts, opinions/cognition, all organs of body, which function or dysfunction as a unit. Hence treatment cannot be focused only on ulcers in colon itself but the treatment plan has to consider patient's make up from childhood, habits, tormenting events of earlier years of life, education, ability to think and reason, etc. along with specific lesions in colon.
Adaptation to such holistic point of view; medical students need to be psychologically, philosophically and socially inclined and trained thereafter; citing the advantages like early success, job satisfaction, minimum professional burned outs, minimum doctor-patient conflicts/ law suits, etc. Following example may clarify the point. $\mathrm{XYZ}$ patient in second consultation asks doctor about the information found on Google. Google mentioned long list of side effects of the medicine prescribed to him, patient reveals. Obviously, patient is worried and scared. Holistic doctor would take patient into confidence by offering patient listening, looking into the matter from patient's point of view, considering his insecurity educate and reassure him/her, providing reliable links to explore Google; unlike any doctor who may tend to be-little patient's concern calling it unnecessary. Awareness, acceptance, adaptation and accommodation of patient's concerns to provide solution are the key features of any holistic doctor.

b) Evolution from "competitiveness" to "accommodativeness": Every "holistic doctor" by virtue of his/her evolution tends to become "accommodative". But this change does not take place automatically. This change occurs when doctor practices the perspective of social interaction. Psycho-Social intelligence helps doctor to be aware of inner dialogue and anticipatory acceptance of inner dialogue of patient, which is similar to Howard Giles 'communication accommodation theory' (CAT), while conversing with patient [10].

Social and psychological inclination helps doctor to become accommodative. Impulsivity and hurry to interact impedes one's journey to be 'accommodative' [11]; however, greatest threat which engulfs impulsivity as well as impatience, to become accommodative is "competitiveness".

Most of the doctors in India experience intense competitiveness. Such competitiveness is necessary to clear examinations but its use is limited up to examinations only. Because competitiveness follows tough mind-setup, inflexibility, indomitable wants to prove oneself. 
Competitiveness when carried forward to prove correctness or superiority of one's stand invariably dissociates doctor from patient. Competitiveness flares up by 'comparison'. Comparison tears apart the bond of compassion, togetherness, unison; thus spoiling doctor-patient relationship. Hence abandoning this very attitude to 'compare' any two things is a hallmark of evolution of doctor.

\section{c) Evolution from "older thinking pattern" to "newer}

thinking pattern": Thinking pattern means the way one thinks. When thinking pattern exists since long and does not change even if situation changes then such pattern of thinking is called older thinking pattern. Opposite to this is a newer thinking pattern; which changes, evolves in response to demands of situation.

Older thinking pattern reflects when one behaves in the same way since many years. Such behavior then becomes repetitive and predictable even when one encounters a new situation. Change in behavior will not take place unless the thinking pattern changes.

This happens because thinking pattern decides the way we all interpret and behave (emotionally, psychologically and physically). Hence if one focuses on behavior-change then change in thinking pattern is inevitable. Thinking pattern is connected with our stored memories, inferences we have derived there-upon to take action.

Major obstacles to change thinking pattern are genes, social-cultural factors. Although, deterrent effect of these obstacles on thinking pattern of any individual cannot be an absolute threat to change one's thinking pattern.

Thinking pattern can be changed with awareness and conscious efforts.

E.g. Couple of angry relatives of a patient when charge at doctor, doctor listens and tries to pacify them (according to older thinking pattern). Doctor cannot run away like a thief but can opt for many other alternatives like at least not facing them alone/without protection, etc. In response to situation if change in behavior takes place which reflects adaption to newer thinking pattern.
Awareness and acceptance of one's own older thinking pattern helps one to change it willingly. This awareness and acceptance would be revealed to oneself when one sits in silence to think meditatively. Deliberate thinking in silence to think meditatively is inevitable and non-negotiable for this evolution. Every doctor should be oriented towards this practice of meditative thinking. T. Grisold., et al. (2017) concludes that mindfulness facilitates unlearning [12]. Change of older thinking pattern is nothing but unlearning and should be adapted with the hopes of future professional gains.

Thus, under the guidance of therapist/guide/self-instructions/ mindfulness all three (a, b, c) above mentioned facets of evolution can be nurtured.

\section{Ulterior objectives}

For friends, family and associates- Speaking ability help avoid friction in between family/friends/associates. Speaking ability facilitates winning hearts of nearer and dearer ones/familiar or unfamiliar individuals.

It helps especially during times of crisis, crisis involving self or others. So, a doctor who has developed fine speaking ability enjoys fine-tuned interpersonal relationship with family and friends.

For patients, relatives - Foremost and striking advantage of speaking ability for any doctor comes handy when death of patient/adverse effects of treatment occurs. After death has struck the blow; conversation based on semantic knowledge is of no use. What matters at that time is the ability to speak effectively and evolved persona of doctor comes handy at that time.

\section{Threats to develop speaking ability}

Negotiation of following four factors helps indirectly to develop speaking ability.

\section{Psychological factors that threaten speaking ability Doctor's mind}

Many a times doctors are in two minds if to tell the patient many details, or would patient/relative understand detailed information. Such dilemma prevails because doctor believes complete disclosure may not actually help patient or may create hassles in decision making process or patient's active or dormant participation in 
decision making process may jeopardize the treatment plan (Mark Lipkin 1979).

This dilemma not only defocuses doctor from what he should convey to patient but disorients him from the desired goal i.e. to win patient's faith and calm down patient's anxiety. This dilemma affect doctor's own faith and patience in a subtler way which can be sensed later [13]; sufficient to create bitter taste in interpersonal communication.

This bitter taste/memory can impede future efforts to enhance speaking ability. Cascade of such events and memory thereof results in either doctor's avoidance behavior or non-beneficial impulsive behavior which ultimately deters doctor to improvise speaking ability.

\section{Undesired comments}

Undesired comments from patient's relatives can initiate negativity in the minds of doctors. Almost every doctor can sure stop such negative self-talk because most of the doctor's are trained to be decisive, a function of frontal lobe.

This concept is corroborated by Kazuo Nishimura., et al. (2012) [14] who detected that decisiveness helps stop thinking. However, those who cannot stop thinking happen to be co-operative, Kazuo Nishimura., et al. observed. So doctors who find difficulty in forgetting undesired comments may evaluate if they are cooperative or not. If doctor is cooperative (submissive) in this particular sense then negativity won't be a hindrance. But most of the doctors are decision-maker by default. And decision makers find it difficult to be cooperative.

So is there a way out? Solution lies in applying a "doctor-patient partnership" method during treatment. Way back in 2008, Margaret Turner-Warwick of Britain spoke about essential need of doctor-patient partnership to be evolved for betterment of health services [15].

When doctor allows patient in the capacity of partner then patient's negative attitude as well as negative comments disappear. Other way round, partnership would facilitate doctor to take and implement decisions rather faster; cutting the time otherwise needed for negotiation. This partnership deters patients/relatives to raise objections as well.

\section{Memory of negative experiences}

Past negative experiences can sure daunt any doctor. Negative memories can be looked upon as a learning lesson if doctor trains himself or herself to be kind and forgiving one. Kindness, forgiveness which is a basis of empathy cannot be implemented unless it is taught in medical school because doctors consider themselves a product of medical education [1].

Kindness and forgiveness are unique soft skills every doctor must adapt. These are imperative and non-negotiable soft skills for every doctor. Similar views are mentioned by Drs. Stephen Trzeciak and Anthony Mazzarelli in their book (2009) 'Compassionomics'. These two skills not only enhances self image of doctor but helps create respectful image in the eyes of patients. This respectful image indirectly helps doctor speak effectively.

\section{Atmosphere in medical schools}

Medical schools traditionally don't facilitate students as well as teachers to respect patients as a whole/as a person. Hence doctors tend not to value communication equal to or more than medical treatment.

\section{Competition in medical education}

Medical education itself is a highly competitive field. Competition here thrives on 'exclusion', e.g. diagnosis or differential diagnosis is maneuvered by method of exclusion. Competition teaches one to exclude and not to include. Exclusion makes concentration better thus facilitates sharpening of the skill.

Sharper the edge of competitiveness blunter becomes the kindness. Thus, respect and kindness for patient cannot be prioritized while treating a patient in the race of competition. This ingrained impression in the minds of budding doctor lasts long enough to exclude communication skill or keep communication skill in infantile stage even in later professional life. Thus, chances of bad communication and creation of bad image in patient's mind increases relentlessly.

Rat-race of competition drives medical students to achieve more marks or higher grades which in turn negatively affect social and emotional intellectual inclination. Interaction with patients who are living and thinking humans heavily rely on social and emotional intellectual understanding. Interaction with patient's com- 
munity influences doctor heavily to either mould or be rigid while responding to patients/relatives. Inability to mould threatens the communication skill. Tendency to mould threatens diagnostic accuracy. This seems like walking on a tight rope. So one wonders; what is a solution?

If communication skills are introduced to medical students while undergoing training of 'history taking' then rigidity of diagnostic ability and flexibility of communication skills can be meshed effectively. If assessment of history taking is valued in examination separately then communication skill will surely be attended by medical students and teachers to resolve the above mentioned riddle. Rebecca Casans and Mithilesh Lal writes views about significance of communication in their book titled 'Communication scenario for the MRCPCH and DCH clinical examination' which supports this view.

\section{Selective communication approach}

Google-doctor-Google patients are experienced by almost all doctors now-a-days. Such patients might irk some clinicians; some may find them difficult to treat with. Such patients invariably interfere or want involvement in decision-making process. Most of the doctors cooperate and employ either paternalistic/shared/ informed method to communicate [16]. Paternalistic is an age old traditional method where all decisions were taken by doctor alone and patients used to obey. This method made doctors de-value importance of communication. Lack of communication resulted in damaged doctor-patient bonding.

To overcome this situation informed decision making was adapted. To some extent and in some patients it was acceptable, but it did not resolve doubts in the minds of some patients. Such patients definitely need to be included in shared decision making method. There by one concludes that every doctor must find out which method suits which patient; execution of most be-fitting method would sure make communication more effective.

\section{Neurological explanation of threat to develop speaking ability}

Various circuits that involve Amygdale-prefrontal cortex, mPFCNucleus Acumbens (NA)-Ventral tagmental area (VTA) of brain stem, Hippocampus -mPFC, PFC-Dorsal Raphe Nucleus (DRN) help in enhancing and maintaining communication.

Functions of PFC can be of great advantage or disadvantage. If one focuses on 'meta-cognitive functions' of PFC, i.e. PFC does error monitoring/reality testing/thinking of our own thinking/learning. PFC does facilitate unlearning-relearning. Thus, one realizes that PFC can be a boon or curse; boon if PFC remains up-regulated and curse if PFC remains down-regulated.

One can check one's status of PFC through one's behavior only.

Hence focus of any doctor need not to be on his/her "healthy thoughts/healthy mind" but should be on "congenial behavior" of self; because behavior/verbal communication of doctor creates impression on self and others. Hence, guide-lines to know the neural status should be drawn from one's own behavior.

Medial Prefrontal cortex (mPFC) through its connectivity is the main part of brain that is responsible for behavior [17].

Neuronal activity in between medial Prefrontal cortex (mPFC) and Amygdala helps 'social-decision making'. This neuronal activity gets suppressed as a result of stress or anxiety provoking events experienced during life. For example, threatening or traumatic experience of communication with patient/care-takers.

Function of mPFC is to regulate emotions and integrate affective state with autonomic and neuro-endocrine stress regulating system. Thus, many a times one responds without much overlay or conscious thoughts; just like as if responding automatically, impulsively in a harsh manner; this indicates impaired or untrained functioning of PFC.

Amygdala and mPFC helps in maintenance of fear extinction as well [18] highlighting problem and solution which sits in PFC and in its neural connectivity; where fearful memories get extinct as a result of re-learning executed by $\mathrm{PFC}$.

Another circuit is in PFC and DRN that plays role to appropriate actions or execute re-learned interaction in such cases.

Serotonin level which lowers as a result of stress threatens speaking ability. Appropriateness of action/speech is determined by Serotonin which is maneuvered by DRN under the control of PFC.

\section{Role of biological faith to develop speaking ability}

Faith facilitates communication and bonding in between doctors and patients. Faith facilitates development of sympathy, empathy, down-regulates 'ego-centric behavior'. 
But many doctors today out of ignorance push aside the importance of developing 'faith'; assuming that faith belongs to the domain of philosophy or spiritual practices. In fact 'faith' is developed biologically and made to function in PFC, Amygdala, and Hippocampus [19]. Neurotransmitters like Serotonin, Dopamine play role in developing faith.

Faith can be biologically developed by prayer, meditation. Meditation increases neural activity in PFC that eventually up-regulates it. Prayer increases activities in anterior cingulate cortex (ACC). Upregulated ACC facilitates compassion, sympathy, and attachment with others (patients in this case). Fear and anger is also subdued by heightened activities in ACC [19].

Hence it should be remembered that faith is a biological entity [19] that can used judicially and wisely to develop bonding which directly or indirectly helps speaking ability.

\section{Breaking bad news (BBN) threat-in-disguise to develop speak-}

ing ability: [20] BBN is inevitable in any doctor's life; not only in oncologist's professional life. Effective communication skills, training, and experience are crucial in BBN. More than $80 \%$ doctors don't have training of BBN.

However, memorization of acronym 'SPIKES' can offer them insight and motivation to BBN. 'S' for Set up/setting up a stage to break the bad news. It's better to have a chamber (stage for BBN) with couple of chairs to accommodate relatives, prior intimation, and uninterrupted atmosphere. ' $\mathrm{P}$ ' for perception of relatives/patients; meaning doctor has to inquire or think meditatively before breaking the news if patients/relatives have some perception of illness and anticipation of bad news. If patients had anticipation of abd news then such stage could facilitate doctor to select sentences while speaking. Newer thinking pattern $(3, \mathrm{~A}, \mathrm{ii}, \mathrm{c}$ above) which is relearned by doctor help to build background before BBN to help patients/relative to cope up with bad news with relative ease. If perception is absent then lot of effective communication becomes mandatory to intimate concerned ones about future consequences of illness i.e. bad news. 'I' stands for information; that should be offered once mental perception is achieved. Broad information about the illness or diagnosis is given first emphasizing that not all information is applicable to the patient in particular. ' $\mathrm{K}$ ' stands for knowledge that is pertinent to patient's illness in holistic perspective. Knowledge may include diagnosis, types of treatment, and re- lationship of various characteristics of patient to his/her disease (e.g. relationship of alcohol overuse and dementia). It also includes pros and cons of treatment and expectations thereof, possible consequences along with possibility of errors in speculation. ' $E$ ' indicates doctor's response to emotions of patient/relatives after BBN. Main objective of ' $\mathrm{E}$ ' is to reinforce and maintain faith of patient/ relatives.

Patient/relatives' response could be shock/silence/anger/ blame/denial/guilt/sadness, etc. 'E' should invariably involve empathy (not sympathy, it can provoke emotional turmoil), kindness and firmness that is delivered in a slow speech. Eye to eye contact is made if possible. Selective use of pause, willingness to get interrupting queries should be executed precisely. Doctor needs to accept and listen patiently to their reactions while BBN. Doctor need not hurry to defend even if circumstances prevail because reactions die down easily if doctor maintains resilient and kind stand. 'S' stands for strategy ahead of BBN. This strategy can be chalked out once relatives/patient come out of impact of BBN.

'SPIKES' cannot be delivered in one sitting only. So, it should be offered on multiple times, executing judiciously and wisely using social intelligence or street-smartness while communicating.

Maneuver of BBN will surely encounter difficulties like denial, non-compliance, deaf ears, misunderstanding, weakening of confidence in treating doctor, patient's denial that doctor had informed them about bad news, legal issues, etc. All doctors need to keep detailed records of $\mathrm{BBN}$ in writing.

Despite these hurdles doctor has to maintain professional dignity, benevolent attitude, duty to care, patient centered approach while executing BBN over a period of time. Many a times BBN makes doctor vulnerable to stress/guilt/negative self-talk/frustration. To survive BBN execution induced burnout, doctor can adapt 'learning attitude' that will protect doctor's mPFC from getting dampened or down regulated.

\section{Methods to develop speaking ability}

Un-learn: Alert cognition of medical practitioners or mere conviction to change speaking style may not be sufficient to develop effective speaking ability, because total unlearning is a myth [21]. Unlearning takes place by re-learning which is called substitution or displacement learning which is equally necessary as that of new- 


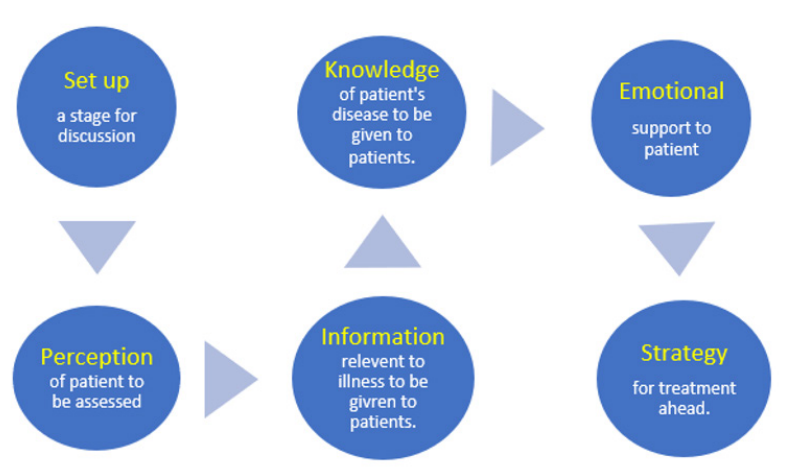

Diagram 1: It is illustrating cascade of 'SPIKES' used in BBN.

learning, to be a successful professional $[12,21]$. Cognitive process with focus to future gains plays role in the process of unlearning, T. Grisold., et al. concludes [12]. How does one unlearn and what should be unlearned? Past memories of non-beneficial communications or interactions with patients/relatives need unlearning by suspending our current thinking pattern [12].

\section{Neuroscience of unlearning}

Unlearning is difficult because it involves anxiety, stress and emotional threat Max Visser had mentioned in January 2017 [22]; especially scary memories are most difficult ones to be washed out/unlearned. Scary memories are products of real-life self-experience or product of experiences of others reflected upon by self. These memories are retained in the form of neural codes or engram/memory traces (Richard Semon 1904).

Human memory is nothing but 'codes'; one can infer from the above paragraph. So, let's affirm now that we need to unlearn 'codes' and that's not going to be very difficult if processed cognitively. Unlearning or relearning takes place either by reflective or cognitive and reflexive or habitual process. Let's use reflective or cognitive process to unlearn bad experiences. T. Grisold., et al. corroborates importance of cognitive process to unlearn [12].

Now the question remains how we unlearn 'particular code'. First very essential step to take to unlearn is to 'accept' fully that our past bad experience is a 'code' in our mind. Let's say for sake of convenience through self-talk that it was a scary code/image indeed. Once one says that it's a scary image; it becomes easy to unlearn it. For example, don't we forget or unlearn scary movies after a day or so under the retext or self-suggestion that it's nothing but an image/neural code. So, acceptance of bad past memories though experienced in real life situations can also be forgotten or unlearned once one accepts it under the pretext of an 'neural code'. Otherwise rejuvenating bad past memories could storm the individual with self-blame, mourning, feelings of insecurity or as if starting a grieving cycle once again [12].

Hence, author believes that acceptance of past memories as 'neural codes' of our mind is the most suitable avenue to unlearn.

But sheer acceptance may not biologically help; forgotten memories or images stand chance to prop up reflexively under the stressful situation in later life. Hence, it is inevitable for all of us to 'like' these scary images.

How to like scary images? Another self-suggestion (cognitive change) may help at this stage. Assume all scary images of nonbeneficial interactions with patients are a 'lessons to learn'.

So, if self-affirmation is made that non-beneficial experiences are a learning lesson then bitterness or fear or stress of those lessons can be forgotten or unlearned; opening the mind eventually to new-learning as against the rejuvenation of habitually learned behavior, that is "older thinking pattern". This exercise biologically takes place in pre-frontal cortex (PFC); remember one of the functions of PFC is 'thinking to think'. As a consequence of this relearning, process of motivation begins in PFC. Neural traffic in between mPFC and Nucleus Acumbens (NA) of dorsal striatum strengthens it. Pre-limbic part of Amygdala (PLA) helps to maintain this relearning.

Thus, one understands that self-suggestion though appears psychological but carves inerasable marks on biological part of the brain to overcome psychological as well as biological threats to effective speaking ability. See the diagram 2 below which creates a visual impression.

\section{Elaboration of point of discussion}

Many a doctors are trained and skilled to say their point in a few words. Similarly, many believe that listeners can grasp subject matter easily without getting confused if information is given in brief. But interestingly researchers do not corroborate so. 


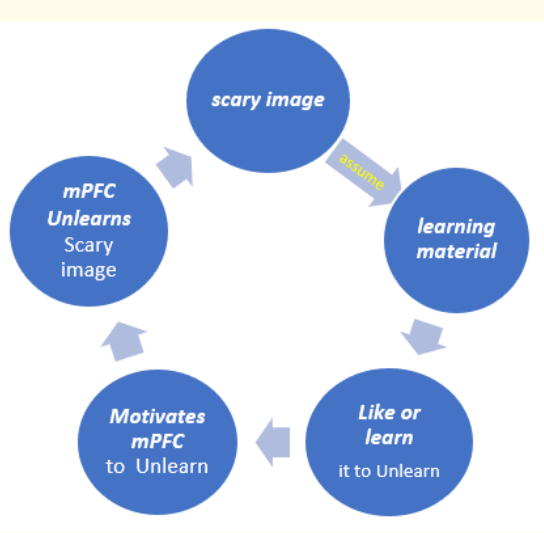

Diagram 2: Five steps to unlearn.

Patients/care-takers need long time to comprehend, internalize or visualize information regarding health/disease.

Brief case report: Recently a patient of known psychiatric illness was attended in psychiatric OPD complaining of severe weakness, extreme fatigability, along with other complaints. Primitive examination itself revealed that $\mathrm{SPO}_{2}$ of patient was $46 \%$; other clinical findings and history too indicated possibility of Covid 19 infection and potentially looming fatality. Care-taker of patient could not believe it because of unexpected nature of news and also because psychiatrist (not a Covid 19 specialist or physician) is breaking the bad news. Un-expectation/shock pushed care-taker in 'denial' mode. In this case information could not be delivered in a few words.

Detail elaboration of findings and possible consequences need to be communicated to care-taker in a sympathetic and caring manner. Doctor's pitch/tone of voice, facial expressions, slow paced speech, reassuring gesture and patient dialogue are in exquisite demand while communicating under such scenario; whereas brief monologue could sure turn care-takers against the doctor.

So, it is imperative while offering information doctor need to adapt "patient-centered approach" and avoid treatment centered approach. While adapting "patient centered approach" doctor allows patient/care-takers to participate in decision making process. This approach reinforces doctor-patient bonding, makes communication effective and rewarding. Advantages of this approach weigh more than that of disadvantages like delay in taking decision or excessive consumption of time.

Brief case report: 40 year old female patient with rural background and illiteracy was attended in a psychiatry opd. Her diagnosis was major depressive disorder with severe anxiety with somatic delusion (mood congruent psychosis). Stressor was her dilation and curettage (D and $\mathrm{C}$ ) procedure conducted by gynaecologist 6 months before.

History narrated by both patient and her husband revealed that patient had ambiguous complaints around pelvic region along with infrequent noxious experience of vagal discharge since some time. On their own they decided to get USG done. Which was available in near by facility. Radiologist who conducted USG advised them to consult a gynaecologist; so they did. Gynaecologist advised D and C. Patient was very anxious and unwilling to undergo $\mathrm{D}$ and $\mathrm{C}$ due to her own fear of surgical intervention through vagina. But husband and gynaecologist insisted upon; so she consented though unwilling and fearful.

3 to 4 days after her D and C she developed extreme anxiety and her negativity regarding the procedure increased multifold. As a result of new complaints husband-wife duo revisited gynaecologist. Her symptoms of severe anxiety like pacing up and down, restlessness, uneasiness, expression of displeasure, disturbed sleep, fearfulness, other depressive features, persisted even after treatment for 6 long months from different doctors including gynaecologist. Her major concern was that her vagina or region around vagina had become extremely weak or as if vagina is sinking inside.

This case reveals that patient's biased mal-formed cognition might not have been attended or amended before D and C (Author's speculation). Purpose and effects of D and C might not have been (author's speculation) adequately explained before in a way to convince patient that $\mathrm{D}$ and $\mathrm{C}$ is a trivial procedure; in other words, psycho-education regarding adverse effects or benefits might not have been explained to patient and her husband in order to pacify her pre-existing anxiety [13]. This case may highlight on non-alignment of patient's expectations and that of treating physician's behavior [4]. This case could be an example of 'ineffective speaking' that might be considered as an attributing factors behind consistent surge of anxious state enough to provoke psychosis. 


\section{Conclusion}

Doctor's attitude towards communication or ability to speak effectively with patients/care-takers makes the whole difference. If doctor considers 'communication' with patients/care-takers a great opportunity to 'succeed' without legal cases in professional career then communication sure reaps benefits not only for patients but for doctors as well. Doctors must understand that effective speaking leads to professional success.

Non-compliant patients of chronic diseases, inflated expenditure of treatment, impending death of patient, expected partial recovery, unavoidable long list of medicines/investigations, relapsing nature of symptoms, residual symptoms after surgery, potential complaints in consumer courts [23], etc. are the few examples where every doctor, willing or not willing to communicate, realizes compulsory need of effective speaking ability.

\section{Conflict of Interest}

None.

\section{Acknowledgement}

1. I want to acknowledge the contribution of Dr. Sanjeev Saoji for pre publication review. Dr. Sanjeev Saoji is Prof and HOD Dept of Psychiatry, JIIU's IIMSR Medical College, Badlapur, Jalna, Maharashtra, India.

2. I wish to thank Dr. Deepika Chandhok, MDS, Endodontics, Associate Professor VSPM Dental College, Nagpur and President Nagpur medico, Junior Chamber (Jaycee), India for inviting me to speak to medical students on this subject.

\section{Bibliography}

1. Kerasidou A and Horn R. "Making space for empathy: supporting doctors in the emotional labour of clinical care". BMC Medical Ethics 17 (2016): 8.

2. Susan E Heckler and Terry L Childers. "Hemispheric Lateralization: the Relationship of Processing Orientation With Judgement and Recall Measures For Print Advertisements", in NA - Advances in Consumer Research Volume 14, editions. Melanie Wallendorf and Paul Anderson, Provo, UT: Association for Consumer Research, Pages (1987): 46-50.

3. Ortigue S., et al. "Right hemisphere dominance for understanding the intentions of others: evidence from a split-brain patient". BMJ Case Report (2009): bcr07.2008.0593.

4. Odero A., et al. "Core Values that Influence the PatientHealthcare Professional Power Dynamic: Steering Interaction towards Partnership". International Journal of Environmental Research and Public Health 17.22 (2020): 8458.

5. Baktir Hasan. "Speech Act Theory; Austin and Searle: Derrida's Response And Deleuze's Theory Of Order-Word". Language, Literature and Cultural Studies. V (2012): 202-211.

6. Ha JF and Longnecker N. "Doctor-patient communication: a review". Ochsner Journal 10.1 (2010): 38-43.

7. Hellström 0. "The importance of a holistic concept of health for health care. Examples from the clinic". Theoretical Medicine and Bioethics 14.4 (1993): 325-342.

8. Borins M. "Holistic medicine in family practice". Canadian Family Physician 30 (1984): 101-106.

9. Malik RF., et al. "Do 'physicians in the lead' support a holistic healthcare delivery approach? A qualitative analysis of stakeholders' perspectives". BMJ Open 8 (2018): e020739.

10. Chevalier BAM., et al. "Investigating strategies used by hospital pharmacists to effectively communicate with patients during medication counselling". Health Expect 20.5 (2017): 1121-1132.

11. Yovetich Nancy A., et al. "Accommodative Behavior in Close Relationships: Exploring Transformation of Motivation". Journal of Experimental Social Psychology 30.2 (1994): 138-164.

12. T Grisold., et al. "Unlearning before Creating new Knowledge: A Cognitive Process, Conference: Hawaii International Conference on System Sciences (2017).

13. Chaturvedi SK. "Truth telling and communication skills". Indian Journal of Psychiatry 51.3 (2009): 227.

14. Kazuo Nishimura., et al. "Thinking Patterns, Brain Activity and Strategy Choice". Journal of Physics: Conference Series (2012): 344012004.

15. Margaret Turner-Warwick. "The patient-doctor partnership over 60 years and the role of the royal medical colleges". Clinical Medicine 8.6 (2008). 
16. Charles C., et al. "Decision-making in the physician-patient encounter: revisiting the shared treatment decision-making model". Social Science and Medicine 49.5 (1999): 651-661.

17. Franklin T., et al. "Prefrontal cortical control of a brainstem social behavior circuit". Nature Neuroscience 20 (2017): 260270.

18. Akirav I and Maroun M. "The role of the medial prefrontal cortex-amygdala circuit in stress effects on the extinction of fear". Neural Plasticity (2007): 30873.

19. Madhav Raje. "Is Faith An Imagination? A Brief Commentary, Paripex". Indian Journal of Research 8.2 (2019): 2250-1991.

20. Walter F Baile., et al. "Spikes-A Six-Step Protocol for Delivering Bad News: Application to the Patient with Cancer". The Oncologist 5 (2000): 302-311.

21. R Rushmer and H T O Davies. "Unlearning in health care". Quality and Safety in Health Care 13.2 (2004): ii10-ii15.

22. Max Visser. "Learning and unlearning: a conceptual note". The Learning Organization 24.1 (2017): 49-57.

23. Pallavi Bradshaw. "Good communication reduces risk of a complaint or claim". British Medical Journal 367 (2019): 16160.

Volume 4 Issue 11 November 2021

(C) All rights are reserved by Madhav Gajananrao Raje. 